\title{
Graphical approximation of common solutions to generalized nonlinear relaxed cocoercive operator equation systems with $(A, \eta)$-accretive mappings
}

\author{
Fang $\mathrm{Li}^{1}$, Heng-you Lan ${ }^{*}$ and Yeol Je Cho ${ }^{2}$
}

\author{
* Correspondence: \\ hengyoulan@163.com \\ 'Department of Mathematics, \\ Sichuan University of Science and \\ Engineering, Zigong, 643000 \\ Sichuan, People's Republic of China \\ Full list of author information is \\ available at the end of the article
}

\begin{abstract}
In this paper, we develop a new perturbed iterative algorithm framework with errors based on the variational graphical convergence of operator sequences with $(A, \eta)$ accretive mappings in Banach space. By using the generalized resolvent operator technique associated with $(A, \eta)$-accretive mappings, we also prove the existence of solutions for a class of generalized nonlinear relaxed cocoercive operator equation systems and the variational convergence of the sequence generated by the perturbed iterative algorithm in q-uniformly smooth Banach spaces. The obtained results improve and generalize some well-known results in recent literatures.
\end{abstract} 2000 Mathematics Subject Classification: 47H05; $49 \mathrm{~J} 40$

Keywords: $(A, \eta)$-accretive mapping, Generalized resolvent operator technique, Generalized nonlinear relaxed cocoercive operator equation systems, New perturbed iterative algorithm with errors, Variational graphical convergence

\section{Introduction}

It is well known that standard Yosida regularizations/approximations have been tremendously effective to approximation solvability of general variational inclusion problems in the context of resolvent operators that turned out to be nonexpansive. This class of nonlinear Yosida approximations have been applied to approximation solvability of nonlinear inhomogeneous evolution inclusions of the form

$$
f(t) \in u^{\prime}(t)+M u(t)-\omega u(t), \quad u(0)=u_{0}
$$

for almost all $t \in[0, T]$, where $T \in(0,1)$ is fixed, $\omega \in R$ (see [1]). For more general details on approximation solvability of general nonlinear inclusion problems, we refer the reader to [2-18] and the references therein.

On the other hand, it is well known that variational inequalities and variational inclusions provide mathematical models to some problems arising in economics, mechanics, and engineering science and have been studied extensively. There are many methods to find solutions of variational inequality and variational inclusion problems. Among these methods, the resolvent operator technique is very important. For some literature, we recommend to the following example, and the reader $[2-15,17,18]$ and the references therein.

(c) 2012 Li et al; licensee Springer. This is an Open Access article distributed under the terms of the Creative Commons Attribution License (http://creativecommons.org/licenses/by/2.0), which permits unrestricted use, distribution, and reproduction in any medium, provided the original work is properly cited. 
Example 1.1. ([19]) Let $V: R^{n} \rightarrow R$ be a local Lipschitz continuous function, and let $K$ be a closed convex set in $R^{n}$. If $x^{*}$ is a solution to the following problem:

$$
\min _{x \in K} V(x),
$$

then

$$
0 \in \partial V\left(x^{*}\right)+\mathcal{N}_{K}\left(x^{*}\right)
$$

where $\partial V\left(x^{*}\right)$ denotes the subdifferential of $V$ at $x^{*}$ and $\mathcal{N}_{K}\left(x^{*}\right)$ the normal cone of $K$ at $x^{*}$.

In 2006, Lan et al. [7] introduced a new concept of $(A, \eta)$-accretive mappings, which provides a unifying framework for maximal monotone operators, $m$-accretive operators, $\eta$-subdifferential operators, maximal $\eta$-monotone operators, $H$-monotone operators, generalized $m$-accretive mappings, $H$-accretive operators, $(H, \eta)$-monotone operators, and $A$-monotone mappings. Recently, by using the concept of $(A, \eta)$-accretive mappings and the resolvent operator technique associated with $(A, \eta)$-accretive mappings, Jin [5] introduced and studied a new class of nonlinear variational inclusion systems with $(A, \eta)$-accretive mappings in $q$-uniformly smooth Banach spaces and developed some new iterative algorithms to approximate the solutions of the mentioned nonlinear variational inclusion systems. Furthermore, by using the resolvent operator technique, Petrot [14] studied the common solutions for a generalized system of relaxed cocoercive mixed variational inequality problems and fixed point problems for Lipschitz mappings in Hilbert spaces, and Agarwal and Verma [2] introduced and studied a new system of nonlinear (set-valued) variational inclusions involving $(A, \eta$ )-maximal relaxed monotone and relative $(A, \eta)$-maximal monotone mappings in Hilbert spaces and proved its approximation solvability based on the variational graphical convergence of operator sequences. For more literature, we recommend to the reader $[9,20]$ and the references therein.

Motivated and inspired by the above works, the purpose of this paper is to consider and study the following generalized nonlinear operator equation system with $(A, \eta$ )-accretive mappings in real Banach space $\mathcal{B}_{1} \times \mathcal{B}_{2}$ :

Find $(x, y) \in \mathcal{B}_{1} \times \mathcal{B}_{2}$ and $u \in S(x), v \in T(y)$ such that

$$
\left\{\begin{array}{l}
p(x)=R_{\eta_{1}, M_{1}(\cdot, x)}^{\rho \lambda_{1}, A_{1}}\left[\left(1-\lambda_{1}\right) A_{1}(p(x))+\lambda_{1}\left(A_{1}(f(y))-\rho N_{1}(u, y)+a\right)\right], \\
h(y)=R_{\eta_{2}, M_{2}(\gamma, \cdot)}^{\rho_{2}, M_{2}}\left[\left(1-\lambda_{2}\right) A_{2}(h(y))+\lambda_{2}\left(A_{2}(g(x))-\varrho N_{2}(x, v)+b\right)\right],
\end{array}\right.
$$

where for all $\quad(x, y) \in \mathcal{B}_{1} \times \mathcal{B}_{2}, R_{\eta_{1}, M_{1}(\cdot, x)}^{\rho \lambda_{1}, A_{1}}=\left(A_{1}+\rho \lambda_{1} M_{1}(\cdot, x)\right)^{-1} \quad$ and $R_{\eta_{2}, M_{2}\left(y_{1},\right)}^{\varrho \lambda_{2}, A_{2}}=\left(A_{2}+\varrho \lambda_{2} M_{2}\left(y_{1} \cdot\right)\right)^{-1}$ are two resolvent operators and two constants $\begin{aligned} \rho, \varrho>0, N_{1}: \mathcal{B}_{1} \times \mathcal{B}_{2} & \rightarrow \mathcal{B}_{1}, N_{2}: \mathcal{B}_{1} \times \mathcal{B}_{2} \rightarrow \mathcal{B}_{2}, p: \mathcal{B}_{1} \rightarrow \mathcal{B}_{1}, h: \mathcal{B}_{2} \rightarrow \mathcal{B}_{2}, f: \text { are single- } \\ \mathcal{B}_{2} & \rightarrow \mathcal{B}_{1}, g: \mathcal{B}_{1} \rightarrow \mathcal{B}_{2}\end{aligned}$

valued operators, $\lambda_{1}, \lambda_{2}>0$ are two constants, $(a, b) \in \mathcal{B}_{1} \times \mathcal{B}_{2}$ is an any given element, and $S: \mathcal{B}_{1} \rightarrow 2^{\mathcal{B}_{1}}, T: \mathcal{B}_{2} \rightarrow 2^{\mathcal{B}_{2}}, A_{i}: \mathcal{B}_{i} \rightarrow \mathcal{B}_{i}, \eta_{i}: \mathcal{B}_{i} \times \mathcal{B}_{i} \rightarrow \mathcal{B}_{i}, M_{i}: \mathcal{B}_{i} \times \mathcal{B}_{i} \rightarrow 2^{\mathcal{B}_{i}}(i=1,2)$ are any nonlinear operators such that for all $x \in \mathcal{B}_{1}, M_{1}(\cdot, x): \mathcal{B}_{1} \rightarrow 2^{\mathcal{B}_{1}}$ is an $\left(A_{1}, \eta_{1}\right)$ accretive mapping and $M_{2}\left(y_{1} \cdot\right): \mathcal{B}_{2} \rightarrow 2^{\mathcal{B}_{1}}$ is an $\left(A_{2}, \eta_{2}\right)$-accretive mapping for all $y \in \mathcal{B}_{2}$, respectively. 
Based on the definition of the resolvent operators associated with $(A, \eta)$-accretive mappings, the Equation (1.1) can be written as

$$
\left\{\begin{array}{l}
a \in A_{1}(p(x))-A_{1}(f(y))+\rho N_{1}(u, y)+\rho M_{1}(p(x), x) \\
b \in A_{2}(h(y))-A_{2}(g(x))+\varrho N_{2}(x, v)+\varrho M_{2}(y, h(y))
\end{array}\right.
$$

Remark 1.1. For appropriate and suitable choices of $\mathcal{B}_{i}, A_{i}, \eta_{i}, N_{i}, M_{i}(i=1,2), p, h, f, g, S, T$, one can obtain a number (systems) of quasivariational inclusions, generalized (random) quasi- variational inclusions, quasi-variational inequalities, and implicit quasi-variational inequalities as special cases of the Equation (1.1) (or problem (1.2)) include. Below are some special cases of problem.

Example 1.2. If $\mathcal{B}_{i}=\mathcal{B}(i=1,2), p=f=h=g, N_{1}(x, \cdot)=N_{2}(\cdot, y)=N(\cdot)$ and $M_{1}(\cdot, x)=$ $M_{1}(\cdot), M_{2}(y, \cdot),=M_{2}(\cdot)$ for all $(x, y) \in \mathcal{B}_{1} \times \mathcal{B}_{2}$ and $a=b=0$, then the problem (1.2) collapses to the following nonlinear variational inclusion system with $(A, \eta)$-accretive mappings:

$$
\left\{\begin{array}{l}
0 \in A_{1}(g(x))-A_{1}(g(y))+\rho N(\gamma)+\rho M_{1}(g(x)) \\
0 \in A_{2}(g(y))-A_{2}(g(x))+\varrho N(x)+\varrho M_{2}(g(y))
\end{array}\right.
$$

The system (1.3) was introduced and studied by Jin [5]. Further, when $A_{i}=A, M_{i}=$ $M(i=1,2)$ and $y=x$, the system (1.3) reduces to a nonlinear variational inclusion of find $x \in \mathcal{B}$ such that

$$
0 \in N(x)+M(g(x))
$$

which contains the variational inclusions with $H$-monotone operator, $H$-accretive mappings, or $A$-maximal ( $m$ )-relaxed monotone (AMRM) mappings in [2,3] as special cases.

Example 1.3. If $\mathcal{B}_{i}=\mathcal{H}(i=1,2)$ is a Hilbert space, $a=b=0, S: \mathcal{B}_{1} \rightarrow \mathcal{B}_{1}$ and $T: \mathcal{B}_{2} \rightarrow \mathcal{B}_{2}$ are two single-valued mappings, $p=f=h=g=S=T=I$ is the identity operator and $M_{1}(\cdot, x)=M_{2}(y, \cdot)=M(\cdot)$ for all $(x, y) \in \mathcal{B}_{1} \times \mathcal{B}_{2}$, then the problem (1.2) is equivalent to solve the following nonlinear variational inclusion system with $(A, \eta)$ monotone mappings:

$$
\left\{\begin{array}{l}
0 \in A_{1}(x)-A_{1}(y)+\rho N(y, x)+\rho M(x) \\
0 \in A_{2}(y)-A_{2}(x)+\varrho N(x, y)+\varrho M(y)
\end{array}\right.
$$

The system (1.4) was introduced and studied by Wang and Wu [18] and contains the generalized system for mixed variational inequalities with maximal monotone operators in [14] as special cases. Moreover, taking $y=x$, then the system (1.4) reduces to finding an element $x \in \mathcal{H}$ such that

$$
0 \in N(x, x)+M(x),
$$

which was considered by Verma [17].

\section{Example}

1.4 .

When $\mathcal{B}_{i}=\mathcal{H}, \lambda_{i}=1(i=1,2), p=h, A_{1}=A_{2}=I, N_{1}(x, \cdot)=N_{2}(\cdot, y)=N(\cdot)$ and $M_{1}(\cdot, x)=M_{1}$ $(\cdot), N_{2}(y, \cdot)=M_{2}(\cdot)$ for all $(x, y) \in \mathcal{B}_{1} \times \mathcal{B}_{2}$, the system (1.1) becomes to the following nonlinear operator equation systems: Finding $(x, y) \in \mathcal{H} \times \mathcal{H}$ such that 


$$
\left\{\begin{array}{l}
h(x)=J_{M_{1}}^{\rho}[f(y)-\rho N(y)] \\
h(y)=J_{M_{2}}^{\rho}[g(x)-\varrho N(x)]
\end{array}\right.
$$

where $J_{M_{1}}^{\rho}=\left(I+\rho M_{1}\right)^{-1}$ and $J_{M_{2}}^{\varrho}=\left(I+\varrho M_{2}\right)^{-1}$. Based on the definition of the resolvent operators, we know that the system (1.5) is equivalent to solve the following system of general variational inclusions:

$$
\left\{\begin{array}{l}
0 \in h(x)-f(y)+\rho N(y)+\rho M_{1}(h(x)) \\
0 \in h(y)-g(x)+\varrho N(x)+\varrho M_{2}(h(y))
\end{array}\right.
$$

which was studied by Noor et al. [12] when $M_{i}=M$ is maximal monotone for $i=1$, 2. Moreover, some special cases of the problem (1.6) can be found in $[4,6]$ and the references therein.

We also construct a new perturbed iterative algorithm framework with errors based on the variational graphical convergence of operator sequences with $(A, \eta)$-accretive mappings in Banach space for approximating the solutions of the nonlinear equation system (1.1) in smooth Banach spaces and prove the existence of solutions and the variational convergence of the sequence generated by the perturbed iterative algorithm in $q$-uniformly smooth Banach spaces. The results present in this paper improve and generalize the corresponding results of $[2,3,5,12,14,17,18]$ and many other recent works.

\section{Preliminaries}

Let $\mathcal{B}$ be a real Banach space with dual space $\mathcal{B}^{*},\langle\cdot, \cdot\rangle$ be the dual pair between $\mathcal{B}$ and $\mathcal{B}^{*}, C B(\mathcal{B})$ denote the family of all nonempty closed bounded subsets of $\mathcal{B}$, and $2^{\mathcal{B}}$ denote the family of all the nonempty subsets of $\mathcal{B}$. The generalized duality mapping $J_{q}: \mathcal{B} \rightarrow 2^{\mathcal{B} *}$ is defined by

$$
J_{q}(x)=\left\{f^{*} \in \mathcal{B}^{*}:\left\langle x, f^{*}\right\rangle=\|x\|^{q},\left\|f^{*}\right\|=\|x\|^{q-1}\right\}, \quad \forall x \in \mathcal{B},
$$

where $q>1$ is a constant. In particular, $J_{2}$ is the usual normalized duality mapping. It is known that, in general, $J_{q}(x)=\|x\|^{q-2} J_{2}(x)$ for all $x \neq 0$, and $J_{q}$ is single-valued if $\mathcal{B}^{*}$ is strictly convex. In the sequel, we always suppose that $\mathcal{B}$ is a real Banach space such that $J_{q}$ is single-valued and $\mathcal{H}$ is a Hilbert space. If $\mathcal{B}=\mathcal{H}$, then $J_{2}$ becomes the identity mapping on $\mathcal{H}$.

The modulus of smoothness of $\mathcal{B}$ is the function $\mathcal{X}_{\mathcal{B}}:[0, \infty) \rightarrow[0, \infty)$ defined by

$$
\mathcal{X}_{\mathcal{B}}(t)=\sup \left\{\frac{1}{2}(\|x+y\|+\|x-y\|)-1:\|x\| \leq 1,\|y\| \leq t\right\} .
$$

A Banach space $\mathcal{B}$ is called uniformly smooth if $\lim _{t \rightarrow 0} \frac{\mathcal{X}_{\mathcal{B}}(t)}{t}=0$.

$\mathcal{B}$ is called $q$-uniformly smooth if there exists a constant $c>0$ such that $\mathcal{X}_{\mathcal{B}}(t) \leq c t^{q}, \quad q>1$. Remark that $J_{q}$ is single-valued if $\mathcal{B}$ is uniformly smooth. In the study of characteristic inequalities in $q$-uniformly smooth Banach spaces, $\mathrm{Xu}$ [21] proved the following result:

Lemma 2.1. Let $\mathcal{B}$ be a real uniformly smooth Banach space. Then, $\mathcal{B}$ is $q$-uniformly smooth if and only if there exists a constant $c_{q}>0$ such that for all $x, y \in \mathcal{B}$, 


$$
\|x+y\|^{q} \leq\|x\|^{q}+q\left\langle y_{1} J_{q}(x)\right\rangle+c_{q}\|y\|^{q} .
$$

In the sequel, we give some concept and lemmas needed later.

Definition 2.1. Let $\mathcal{B}$ be a $q$-uniformly smooth Banach space and $T, A: \mathcal{B} \rightarrow \mathcal{B}$ be two single-valued mappings. $T$ is said to be

(i) accretive if

$$
\left\langle T(x)-T(y), J_{q}(x-y)\right\rangle \geq 0, \quad \forall x, y \in \mathcal{B} ;
$$

(ii) strictly accretive if $T$ is accretive and

$$
\left\langle T(x)-T(y), J_{q}(x-y)\right\rangle=0
$$

if and only if $x=y$;

(iii) $r$-strongly accretive if there exists a constant $r>0$ such that

$$
\left\langle T(x)-T(y), J_{q}(x-y)\right\rangle \geq r\|x-y\|^{q}, \quad \forall x, y \in \mathcal{B} ;
$$

(iv) $\gamma$-strongly accretive with respect to $A$ if there exists a constant $\gamma>0$ such that

$$
\left\langle T(x)-T(y), J_{q}(A(x)-A(y))\right\rangle \geq \gamma\|x-y\|^{q}, \quad \forall x, y \in \mathcal{B} ;
$$

(v) $m$-relaxed cocoercive with respect to $A$ if, there exists a constant $m>0$ such that

$$
\left\langle T(x)-T(y), J_{q}(A(x)-A(y))\right\rangle \geq-m\|T(x)-T(y)\|^{q}, \quad \forall x, y \in \mathcal{B} ;
$$

(vi) $(\pi, \iota)$-relaxed cocoercive with respect to $A$ if, there exist constants $\pi, \iota>0$ such that

$$
\left\langle T(x)-T(y), J_{q}(A(x)-A(y))\right\rangle \geq-\pi\|x-y\|^{q}+\iota\|T(x)-T(y)\|^{q}, \quad \forall x, y \in \mathcal{B} ;
$$

(vii) $s$-Lipschitz continuous if there exists a constant $s>0$ such that

$$
\|T(x)-T(y)\| \leq s\|x-y\|, \quad \forall x, y \in \mathcal{B} .
$$

In a similar way, we can define (relaxed) cocoercivity and Lipschitz continuity of the operator $N(\cdot, \cdot): \mathcal{B} \times \mathcal{B} \rightarrow \mathcal{B}$ in the first and second arguments.

Remark 2.1. (1) The notion of the cocoercivity is applied in several directions, especially to solving variational inequality problems using the auxiliary problem principle 
and projection methods [16], while the notion of the relaxed cocoercivity is more general than the strong monotonicity as well as cocoercivity. Several classes of relaxed cocoercive variational inequalities and variational inclusions have been studied in [2,5,7-10,12,16-18].

(2) When $\mathcal{B}=\mathcal{H}$, (i)-(iv) of Definition 2.1 reduce to the definitions of monotonicity, strict monotonicity, strong monotonicity, and strong monotonicity with respect to $A$, respectively (see $[3,18])$.

Definition 2.2. A single-valued mapping $\eta: \mathcal{B} \times \mathcal{B} \rightarrow \mathcal{B}$ is said to be $\tau$-Lipschitz continuous if there exists a constant $\tau>0$ such that

$$
\|\eta(x, y)\| \leq \tau\|x-y\|, \quad \forall x, y \in \mathcal{B} .
$$

Definition 2.3. Let $\mathcal{B}$ be a $q$-uniformly smooth Banach space, $\eta: \mathcal{B} \times \mathcal{B} \rightarrow \mathcal{B}$ and $A, H: \mathcal{B} \rightarrow \mathcal{B}$ be single-valued mappings. Then set-valued mapping $M: \mathcal{B} \rightarrow 2^{\mathcal{B}}$ is said to be

(i) $\eta$-accretive if

$$
\left\langle u-v, J_{q}(\eta(x, y))\right\rangle \geq 0, \quad \forall x, y \in \mathcal{B}, u \in M(x), v \in M(y) ;
$$

(ii) $r$-strongly $\eta$-accretive if there exists a constant $r>0$ such that

$$
\left\langle u-v, J_{q}(\eta(x, y))\right\rangle \geq r\|x-y\|^{q}, \quad \forall x, y \in \mathcal{B}, u \in M(x), v \in M(y) ;
$$

(iii) $m$-relaxed $\eta$-accretive if there exists a constant $m>0$ such that

$$
\left\langle u-v, J_{q}(\eta(x, y))\right\rangle \geq-m\|x-y\|^{q}, \quad \forall x, y \in \mathcal{B}, u \in M(x), v \in M(y) ;
$$

(iv) $\xi-\hat{\mathbf{H}}$-Lipschitz continuous, if there exists a constant $\xi>0$ such that

$$
\hat{\mathbf{H}}(M(x), M(y)) \leq \xi\|x-y\|, \quad \forall x, y \in \mathcal{B},
$$

where $\hat{\mathbf{H}}$ is the Hausdorff metric on $C B(\mathcal{B})$;

(v) $(A, \eta)$-accretive if $M$ is $m$-relaxed $\eta$-accretive and $(A+\rho M)(\mathcal{B})=\mathcal{B}$ for every $\rho$ $>0$.

Remark 2.2. The $(A, \eta)$-accretivity generalizes the general $(H, \eta)$-accretivity, $(I, \eta)$ accretivity (so-called generalized $m$-accretivity), $H$-accretivity classical $m$-accretivity ( $A$, $\eta$ )-monotonicity, $A$-monotonicity, $(H, \eta)$-monotonicity, $H$-monotonicity, maximal $\eta$ monotonicity, and classical maximal monotonicity as special cases (see, for example, $[1,7,8,13]$ and the references therein.) 
Definition 2.4. Let $A: \mathcal{B} \rightarrow \mathcal{B}$ be a strictly $\eta$-accretive mapping and $M: \mathcal{B} \rightarrow 2^{\mathcal{B}}$ be an $(A, \eta)$-accretive mapping. The resolvent operator $R_{\eta, M}^{\rho, A}: \mathcal{B} \rightarrow \mathcal{B}$ is defined by:

$$
R_{\eta, M}^{\rho, A}(u)=(A+\rho M)^{-1}(u), \quad \forall u \in \mathcal{B} .
$$

Remark 2.3. The resolvent operators associated with $(A, \eta)$-accretive mappings include as special cases the corresponding resolvent operators associated with $(H, \eta)$ accretive mappings, $(A, \eta)$-monotone operators [8], $(H, \eta)$-monotone operators, $H$ accretive operators, generalized $m$-accretive operators, maximal $\eta$-monotone operators, $H$-monotone operators, $A$-monotone operators, $\eta$-subdifferential operators, the classical $m$-accretive, and maximal monotone operators. See, for example, $[1,7,8,13]$ and the references therein.

Lemma 2.2. ([7]) Let $\mathcal{B}$ be a $q$-uniformly smooth Banach space and $\eta: \mathcal{B} \times \mathcal{B} \rightarrow \mathcal{B}$ be $\tau$-Lipschitz continuous, $A: \mathcal{B} \rightarrow \mathcal{B}$ be a $r$-strongly $\eta$-accretive mapping and $M: \mathcal{B} \rightarrow 2^{\mathcal{B}}$ be an $(A, \eta)$-accretive mapping. Then, the resolvent operator $R_{\eta, M}^{\rho, A}: \mathcal{B} \rightarrow \mathcal{B}$ is $\frac{\tau^{q-1}}{r-\rho m}$-Lipschitz continuous, i.e.,

$$
\left\|R_{\eta, M}^{\rho, A}(x)-R_{\eta, M}^{\rho, A}(y)\right\| \leq \frac{\tau^{q-1}}{r-\rho m}\|x-y\|, \quad \forall x, y \in \mathcal{B},
$$

where $\rho \in\left(0, \frac{r}{m}\right)$ is a constant.

Definition 2.5. Let $M^{n}, M: \mathcal{B} \rightarrow 2^{\mathcal{B}}$ be $(A, \eta)$-accretive mappings on $\mathcal{B}$ for $n=0,1$, $2, \ldots$. Let $A: \mathcal{B} \rightarrow \mathcal{B}$ be $r$-strongly $\eta$-monotone and $\beta$-Lipschitz continuous. The sequence $M^{n}$ is graph-convergent to $M$, denoted $M^{n} \stackrel{A-G}{\longrightarrow} M$, if for every $(x, y) \in$ $\operatorname{graph}(M)$, there exists a sequence $\left(x_{n}, y_{n}\right) \in \operatorname{graph}\left(M^{n}\right)$ such that

$$
x_{n} \rightarrow x, \quad y_{n} \rightarrow y \quad \text { as } n \rightarrow \infty .
$$

Based on Definition 2.6 and Theorem 2.1 in [20], we have the following lemma.

Lemma 2.3. Let $M^{n}, M: \mathcal{B} \rightarrow 2^{\mathcal{B}}$ be $(A, \eta)$-accretive mappings on $\mathcal{B}$ for $n=0,1$, $2, \ldots$. Then, the sequence $M^{n} \stackrel{A-G}{\longrightarrow} M$ if and only if

$$
R_{\eta, M^{n}}^{\rho, A}(x) \rightarrow R_{\eta, M}^{\rho, A}(x), \forall x \in \mathcal{B},
$$

where $R_{\eta, M}^{\rho, A}=\left(A+\rho M^{n}\right)^{-1}, R_{\eta, M}^{\rho, A}=(A+\rho M)^{-1}, \rho>0$ is a constant, and $A: \mathcal{B} \rightarrow \mathcal{B}$ is $r$-strongly $\eta$-monotone and $\beta$-Lipschitz continuous.

\section{Algorithms and graphical convergence}

In this section, by using resolvent operator technique associated with $(A, \eta)$-accretive mappings, we shall develop a new perturbed iterative algorithm framework with errors for solving the nonlinear operator equation system $(1.1)$ with $(A, \eta)$-accretive mappings and relaxed cocoercive operators and prove the existence of solutions and the variational convergence of the sequence generated by the perturbed iterative algorithm in $q$-uniformly smooth Banach spaces. 
Above all, we note that the equalities (1.1) can be written as

$$
\left\{\begin{array}{l}
p(x)=R_{\eta_{1}, M_{1}(\cdot, x)}^{\rho \lambda_{1}, A_{1}}(s) \\
s=\left(1-\lambda_{1}\right) A_{1}(p(x))+\lambda_{1}\left(A_{1}(f(y))-\rho N_{1}(u, y)+a\right) \\
h(y)=R_{\eta_{2}, M_{2}(y, \cdot)}^{\varrho \lambda_{2} A_{2}}(t) \\
t=\left(1-\lambda_{2}\right) A_{2}(h(y))+\lambda_{2}\left(A_{2}(g(x))-\varrho N_{2}(x, v)+b\right)
\end{array}\right.
$$

where $\rho, \lambda>0$ are constants. This formulation allows us to construct the following perturbed iterative algorithm framework with errors.

Algorithm 3.1. Step 1. For an arbitrary initial point $\left(x_{0}, y_{0}\right) \in \mathcal{B}_{1} \times \mathcal{B}_{2}$, take $u_{0} \in S$ $\left(x_{0}\right)$ and $v_{0} \in T\left(y_{0}\right)$.

Step 2. Choose sequences $\left\{d_{n}\right\} \subset \mathcal{B}_{1}$ and $\left\{e_{n}\right\} \subset \mathcal{B}_{2}$ are two error sequences to take into account a possible inexact computation of the operator points, which satisfy the following conditions:

$$
\lim _{n \rightarrow \infty} d^{n}=\lim _{n \rightarrow \infty} e^{n}=0, \sum_{n=1}^{\infty}\left(\left\|d^{n}-d^{n-1}\right\|+\left\|e^{n}-e^{n-1}\right\|\right)<\infty .
$$

Step 3 . Let the sequence $\left\{\left(s_{n}, t_{n}, x_{n}, y_{n}\right)\right\} \subset \mathcal{B}_{1} \times \mathcal{B}_{2} \times \mathcal{B}_{1} \times \mathcal{B}_{2}$ satisfy

$$
\left\{\begin{array}{l}
s_{n}=\left(1-\lambda_{1}\right) A_{1}\left(p\left(x_{n}\right)\right)+\lambda_{1}\left(A_{1}\left(f\left(y_{n}\right)\right)-\rho N_{1}\left(u_{n}, y_{n}\right)+a\right), \\
t_{n}=\left(1-\lambda_{2}\right) A_{2}\left(h\left(y_{n}\right)\right)+\lambda_{2}\left(A_{2}\left(g\left(x_{n}\right)\right)-\varrho N_{2}\left(x_{n}, v_{n}\right)+b\right) \\
x_{n+1}=(1-k) x_{n}+k\left\{x_{n}-p\left(x_{n}\right)+R_{\eta_{1}, M_{1}^{n} \cdot\left(\cdot, x_{n}\right)}^{\rho \lambda_{1}, A_{1}}\left(s_{n}\right)\right\}+d_{n} \\
y_{n+1}=(1-\kappa) y_{n}+\kappa\left\{y_{n}-h\left(y_{n}\right)+R_{\eta_{2}, M_{2}^{n}\left(y_{n,}\right)}^{\rho \lambda_{2}, t_{n}}\left(t_{n}\right)\right\}+e_{n}
\end{array}\right.
$$

where $\quad R_{\eta_{1}, M_{1}^{n}(\cdot, x)}^{\rho \lambda_{1}, A_{1}}=\left(A_{1}+\rho \lambda_{1} M_{1}^{n}(\cdot, x)\right)^{-1}, R_{\eta_{2}, M_{2}^{n}\left(\gamma_{1}\right)}^{\varrho \lambda_{2}, A_{2}}=\left(A_{2}+\varrho \lambda_{2} M_{2}^{n}\left(\gamma_{1} \cdot\right)\right)^{-1}, \lambda_{1}, \lambda \lambda_{2}, \rho, \varrho$ are nonnegative constants and $k, \kappa \in(0,1]$ are size constants.

Step 4. Choose $u_{n+1} \in S\left(x_{n+1}\right)$ and $v_{n+1} \in T\left(y_{n+1}\right)$ such that (see [22])

$$
\begin{aligned}
& \left\|u_{n}-u_{n+1}\right\| \leq\left(1+\frac{1}{n+1}\right) \hat{\mathbf{H}}\left(S\left(x_{n}\right), S\left(x_{n+1}\right)\right), \\
& \left\|v_{n}-v_{n+1}\right\| \leq\left(1+\frac{1}{n+1}\right) \hat{\mathbf{H}}\left(T\left(y_{n}\right), T\left(y_{n+1}\right)\right) .
\end{aligned}
$$

Step 5. If $s_{n}, t_{n}, x_{n}, y_{n}, d_{n}$, and $e_{n}$ satisfy (3.1) and (3.2) to sufficient accuracy, stop; otherwise, set $n:=n+1$ and return to Step 2 .

Now, we prove the existence of a solution of problem (1.1) and the convergence of Algorithm 3.1.

Theorem 3.1. For $i=1,2$, let $\mathcal{B}_{i}$ be a $q_{i}$-uniformly smooth Banach space with $q_{i}>1$, $\eta_{i}, A_{i}, M_{i}, N_{i}(i=1,2)$ and $p, h, f, g$ be the same as in the Equation (1.1). Also suppose that the following conditions hold:

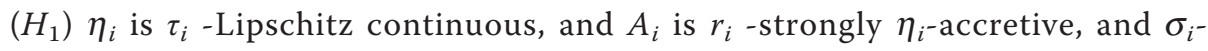
Lipschitz continuous for $i=1,2$, respectively;

$\left(H_{2}\right) p$ is $\delta_{1}$-strongly accretive and $l_{p}$-Lipschitz continuous, $h$ is $\delta_{2}$-strongly accretive and $l_{h}$-Lipschitz continuous, $f$ is $l_{f}$-Lipschitz continuous and $g$ is $l_{g}$-Lipschitz 
continuous, $\quad S: \mathcal{B}_{1} \rightarrow C B\left(\mathcal{B}_{1}\right) \quad$ is $\quad \xi-\hat{\mathbf{H}}$-Lipschitz continuous and $T: \mathcal{B}_{2} \rightarrow C B\left(\mathcal{B}_{2}\right)$ is $\zeta-\hat{\mathbf{H}}$-Lipschitz continuous;

$\left(H_{3}\right) N_{1}$ is $\left(\pi_{1}, \iota_{1}\right)$-relaxed cocoercive with respect to $f_{1}$ and $\varpi_{2}$-Lipschitz continuous in the second argument, and $N_{2}$ is $\left(\pi_{2}, \iota_{2}\right)$-relaxed cocoercive with respect to $g_{2}$ and $\varpi_{1}$-Lipschitz continuous in the first argument, and $N_{1}$ is $\beta_{1}$-Lipschitz continuous in the first variable, and $N_{2}$ is $\beta_{2}$-Lipschitz continuous in the second variable, where $f_{1}: \mathcal{B}_{2} \rightarrow \mathcal{B}_{1}$ is defined by $f_{1}(y)=A_{1} \circ f(y)=A_{1}(f(y))$ for all $y \in \mathcal{B}_{2}$ and $g_{2}: \mathcal{B}_{1} \rightarrow \mathcal{B}_{2}$ is defined by $g_{2}(x)=A_{2} \circ g(x)=A_{2}(g(x))$ for all $x \in \mathcal{B}_{1}$;

$\left(H_{4}\right)$ for $n=0,1,2, \ldots, M_{i}^{n}: \mathcal{B}_{i} \times \mathcal{B}_{i} \rightarrow 2^{\mathcal{B}_{i}}(i=1,2)$ are any nonlinear operators such that for all $x \in \mathcal{B}_{1}, M_{1}^{n}(\cdot, x): \mathcal{B}_{1} \rightarrow 2^{\mathcal{B}_{1}}$ is an $\left(A_{1}, \eta_{1}\right)$-accretive mapping with $M_{1}^{n}(\cdot, x) \stackrel{A_{1}-G M_{1}(\cdot, x)}{\longrightarrow}$, and $M_{2}^{n}\left(y_{1} \cdot\right): \mathcal{B}_{2} \rightarrow 2^{\mathcal{B}_{2}}$ is an $\left(A_{2}, \eta_{2}\right)$-accretive mapping

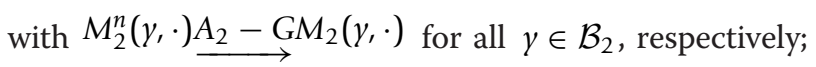

$\left(H_{5}\right)$ there exist constants $v_{i}(i=1,2), \rho \in\left(0, r_{1} / m_{1}\right)$ and $\varrho \in\left(0, r_{2} / m_{2}\right)$ such that

$$
\left\|\begin{array}{l}
R_{\eta_{1}, M_{1}(\cdot, x)}^{\rho \lambda_{1}, A_{1}}(z)-R_{\eta_{1}, M_{1}(\cdot, y)}^{\rho \lambda_{1}, A_{1}}(z) \\
R_{\eta_{2}, M_{2}(x, \cdot)}^{\rho \lambda_{2}, A_{2}}(z)-R_{\eta_{2}, M_{2}(y, \cdot)}^{\rho \lambda_{2}, M_{2}}(z)
\end{array}\right\| \leq v_{2}\|x-y\|, \quad \forall x, y, z \in \mathcal{B}_{1}\|x-y\|, \quad \forall x, y, z \in \mathcal{B}_{2},
$$

and

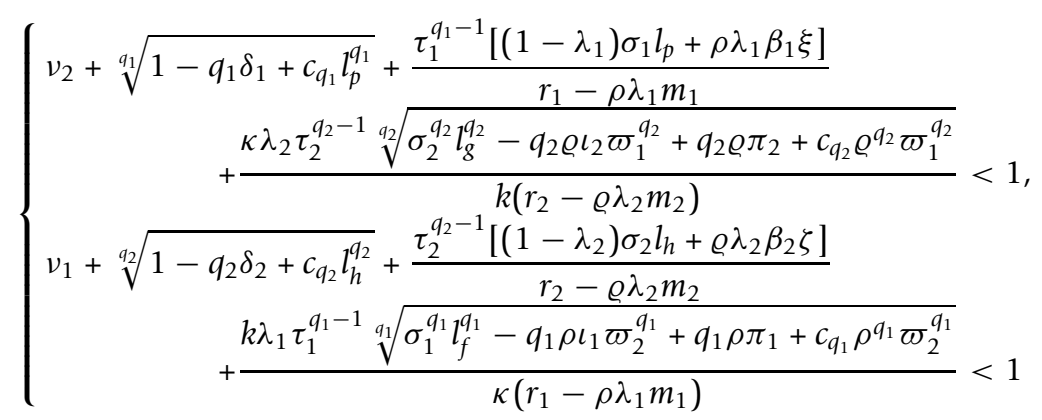

where $c_{q_{1}}, c_{q_{2}}$ are the constants as in Lemma 2.1 and $k, \kappa \in(0,1]$ are size constants.

Then, there exist $\left(x^{*}, y^{*}\right) \in \mathcal{B}_{1} \times \mathcal{B}_{2} u^{*} \in S\left(x^{*}\right), v^{*} \in T\left(y^{*}\right)$ such that $\left(x^{*}, y^{*}, u^{*}, v^{*}\right)$ is a solution of the Equation (1.1) and

$$
x_{n} \rightarrow x^{*}, y_{n} \rightarrow \gamma^{*}, u_{n} \rightarrow u^{*}, v_{n} \rightarrow v^{*}, \quad \text { as } n \rightarrow \infty,
$$

where $\left\{x_{n}\right\},\left\{y_{n}\right\},\left\{u_{n}\right\}$ and $\left\{v_{n}\right\}$ are iterative sequences generated by Algorithm 3.1.

Proof. Define $\|\cdot\| *$ on $\mathcal{B}_{1} \times \mathcal{B}_{2}$ by

$$
\|(x, y)\|_{*}=\|x\|+\|y\|, \quad \forall(x, y) \in \mathcal{B}_{1} \times \mathcal{B}_{2}
$$

It is easy to see that $\left(\mathcal{B}_{1} \times \mathcal{B}_{2},\|\cdot\|_{*}\right)$ is a Banach space. By the assumptions for relaxed cocoercivity and Lipschitz continuity of $N$ with respect to both arguments, strongly accretivity of $p$ and $h$, and Lipschitz continuity of $S, T, p, f, g$ and $h$, Lemmas 2.1 and 2.2, and (3.1)-(3.3), now we know that 


$$
\begin{aligned}
& \left\|N_{1}\left(u_{n}, y_{n-1}\right)-N_{1}\left(u_{n-1}, y_{n-1}\right)\right\| \leq \beta_{1}\left\|u_{n}-u_{n-1}\right\| \\
& \quad \leq \beta_{1}\left(1+n^{-1}\right) \hat{\mathbf{H}}\left(S\left(x_{n}\right), S\left(x_{n-1}\right)\right) \leq \beta_{1} \xi\left(1+n^{-1}\right)\left\|x_{n}-x_{n-1}\right\|, \\
& \left\|A_{1}\left(f\left(y_{n}\right)\right)-A_{1}\left(f\left(y_{n-1}\right)\right)-\rho\left[N_{1}\left(u_{n}, y_{n}\right)-N_{1}\left(u_{n}, y_{n}\right)\right]\right\|^{q_{1}} \\
& \quad \leq\left\|A_{1}\left(f\left(y_{n}\right)\right)-A_{1}\left(f\left(y_{n-1}\right)\right)\right\|^{q_{1}}+\rho^{q_{1}} c_{q_{1}}\left\|N_{1}\left(u_{n}, y_{n}\right)-N_{1}\left(u_{n}, y_{n-1}\right)\right\|^{q_{1}} \\
& \quad-q_{1} \rho\left\langle N_{1}\left(u_{n}, y_{n}\right)-N_{1}\left(u_{n}, y_{n-1}\right), J_{q_{1}}\left(A_{1}\left(f\left(y_{n}\right)\right)-A_{1}\left(f\left(y_{n-1}\right)\right)\right)\right\rangle \\
& \leq \\
& \quad\left(\sigma_{1}^{q_{1}} q_{f}^{q_{1}}-q_{1} \rho l_{1} \varpi_{2}^{q_{1}}+q_{1} \rho \pi_{1}+c_{q_{1}} \rho^{q_{1}} \varpi_{2}^{q_{1}}\right)\left\|y_{n}-y_{n-1}\right\|^{q_{1}}, \\
& \left\|x_{n}-x_{n-1}-\left[p\left(x_{n}\right)-p\left(x_{n-1}\right)\right]\right\| \leq \sqrt[q_{1}]{1-q_{1} \delta_{1}+c_{q_{1}} l_{p}^{q_{1}}}\left\|x_{n}-x_{n-1}\right\|,
\end{aligned}
$$

and

$$
\begin{aligned}
& \left\|s_{n}-s_{n-1}\right\| \\
& =\|\left(1-\lambda_{1}\right) A_{1}\left(p\left(x_{n}\right)\right)+\lambda_{1}\left(A_{1}\left(f\left(y_{n}\right)\right)-\rho N_{1}\left(u_{n}, y_{n}\right)+a\right) \\
& -\left(1-\lambda_{1}\right) A_{1}\left(p\left(x_{n-1}\right)\right)-\lambda_{1}\left(A_{1}\left(f\left(y_{n-1}\right)\right)-\rho N_{1}\left(u_{n-1}, y_{n-1}\right)+a\right) \| \\
& \leq\left(1-\lambda_{1}\right)\left\|A_{1}\left(p\left(x_{n}\right)\right)-A_{1}\left(p\left(x_{n-1}\right)\right)\right\| \\
& +\rho \lambda_{1}\left\|N_{1}\left(u_{n}, y_{n-1}\right)-N_{1}\left(u_{n-1}, y_{n-1}\right)\right\| \\
& +\lambda_{1}\left\|A_{1}\left(f\left(y_{n}\right)\right)-A_{1}\left(f\left(y_{n-1}\right)\right)-\rho\left[N_{1}\left(u_{n}, y_{n}\right)-N_{1}\left(u_{n}, y_{n-1}\right)\right]\right\| \\
& \leq\left[\left(1-\lambda_{1}\right) \sigma_{1} l_{p}+\rho \lambda_{1} \beta_{1} \xi\left(1+n^{-1}\right)\right]\left\|x_{n}-x_{n-1}\right\| \\
& +\lambda_{1} \sqrt[q_{1}]{\sigma_{1}^{q_{1}} l_{f}^{q_{1}}-q_{1} \rho \iota_{1} \varpi_{2}^{q_{1}}+q_{1} \rho \pi_{1}+c_{q_{1}} \rho \rho^{q_{1}} \varpi_{2}^{q_{1}}}\left\|y_{n}-y_{n-1}\right\|, \\
& \left\|x_{n+1}-x_{n}\right\| \\
& \leq(1-k)\left\|x_{n}-x_{n-1}\right\|+k\left\|x_{n}-x_{n-1}-\left[p\left(x_{n}\right)-p\left(x_{n-1}\right)\right]\right\| \\
& +k\left\|R_{\eta 1, M_{1}^{n}\left(\cdot, x_{n}\right)}^{\rho \lambda_{1}, A_{1}}\left(s_{n}\right)-R_{\eta_{1}, M_{1}\left(\cdot, x_{n}\right)}^{\rho \lambda_{1}, A_{1}}\left(s_{n}\right)\right\|+\left\|d_{n}-d_{n-1}\right\| \\
& +k\left\|R_{\eta_{1}, M_{1}\left(\cdot, x_{n}\right)}^{\rho \lambda_{1}, A_{1}}\left(s_{n}\right)-R_{\eta_{1}, M_{1}\left(\cdot, x_{n-1}\right)}^{\rho \lambda_{1}, A_{1}}\left(S_{n}\right)\right\| \\
& +k\left\|R_{\eta_{1}, M_{1}\left(\cdot, x_{n-1}\right)}^{\rho \lambda_{1}\left(A_{1}\right.}\left(s_{n}\right)-R_{\eta_{1}, M_{1}\left(\cdot, x_{n-1}\right)}^{\rho \lambda_{1}, A_{1}}\left(s_{n-1}\right)\right\| \\
& +k\left\|R_{\eta_{1}, M_{1}^{n-1}\left(\cdot, x_{n-1}\right)}^{\rho \lambda_{1} A_{1}}\left(s_{n-1}\right)-R_{\eta_{1}, M_{1}\left(\cdot, x_{n-1}\right)}^{\rho \lambda_{1}, A_{1}}\left(s_{n-1}\right)\right\| \\
& \leq(1-k)\left\|x_{n}-x_{n-1}\right\|+k\left\|x_{n}-x_{n-1}-\left[p\left(x_{n}\right)-p\left(x_{n-1}\right)\right]\right\| \\
& +k v_{2}\left\|x_{n}-x_{n-1}\right\|+\frac{k \tau_{1}^{q_{1}-1}}{r_{1}-\rho \lambda_{1} m_{1}}\left\|s_{n}-s_{n-1}\right\| \\
& +k\left(\varepsilon_{n}+\varepsilon_{n-1}\right)+\left\|d_{n}-d_{n-1}\right\| \\
& \leq\left[1-k\left(1-\theta_{1 n}\right)\right]\left\|x_{n}-x_{n-1}\right\|+k \vartheta_{1}\left\|y_{n}-y_{n-1}\right\| \\
& +k\left(\varepsilon_{n}+\varepsilon_{n-1}\right)+\left\|d_{n}-d_{n-1}\right\|,
\end{aligned}
$$

where $\varepsilon_{l}=\left\|R_{\eta_{1}, M_{1}^{1}\left(\cdot, x_{l}\right)}^{\rho \lambda_{1}, A_{1}}\left(s_{l}\right)-R_{\eta_{1}, M_{1}\left(\cdot, x_{l}\right)}^{\rho \lambda_{1}, A_{1}}\left(s_{l}\right)\right\|$ for $l=n-1, n$ and

$$
\begin{aligned}
\theta_{1, n} & =\nu_{2}+\sqrt[q_{1}]{1-q_{1} \delta_{1}+c_{q_{1}} l_{p}^{q_{1}}}+\frac{\tau_{1}^{q_{1}-1}\left(1-\lambda_{1}\right) \sigma_{1} l_{p}+\rho \lambda_{1} \beta_{1} \xi\left(1+n^{-1}\right)}{r_{1}-\rho \lambda_{1} m_{1}} \\
\vartheta_{1} & =\frac{\lambda_{1} \tau_{1}^{q_{1}-1} \sqrt[q_{1}]{\sigma_{1}^{q_{1}} l_{f}^{q_{1}}-q_{1} \rho \iota_{1} \varpi_{2}^{q_{1}}+q_{1} \rho \pi_{1}+c_{q_{1}} \rho \rho_{1} \varpi_{2}^{q_{1}}}}{r_{1}-\rho \lambda_{1} m_{1}}
\end{aligned}
$$


Similarly, we get

$$
\begin{aligned}
\left\|y_{n+1}-y_{n}\right\| & \leq\left[1-\kappa\left(1-\theta_{2 n}\right)\right]\left\|y_{n}-y_{n-1}\right\|+\kappa \vartheta_{2}\left\|x_{n}-x_{n-1}\right\| \\
& +\kappa\left(\varepsilon_{n}+\varepsilon_{n-1}\right)+\left\|e_{n}-e_{n-1}\right\|,
\end{aligned}
$$

where $\varepsilon_{l}=\left\|R_{\eta_{2}, M_{2}^{l}(y l, \cdot)}^{Q \lambda_{2}, A_{2}}\left(t_{l}\right)-R_{\eta_{2}, M_{2}\left(y_{l} \cdot\right)}^{Q \lambda_{2}, A_{2}}\left(t_{l}\right)\right\|$ for $l=n-1, n$ and

$$
\begin{aligned}
\theta_{2, n} & =v+\sqrt[q_{2}]{1-q_{2} \delta_{2}+c_{q_{2}} l_{h}^{q_{2}}}+\frac{\tau_{2}^{q_{2}-1}\left[\left(1-\lambda_{2}\right) \sigma_{2} l_{h}+\varrho \lambda_{2} \beta_{2} \zeta\left(1+n^{-1}\right)\right]}{r_{2}-\varrho \lambda_{2 m 2}} \\
\vartheta_{2} & =\frac{\lambda_{2} \tau_{2}^{q_{2}-1} \sqrt[q_{2}]{\sigma_{2}^{q_{2}} l_{g}^{q_{2}}-q_{2} \varrho \iota_{2} \varpi_{1}^{q_{2}}+q_{2} \varrho \pi_{2}+c_{q_{2}} \varrho \varrho^{q_{2}} \varpi_{1}^{q_{2}}}}{r_{2}-\varrho \lambda_{2} m_{2}} .
\end{aligned}
$$

follows from (3.5) and (3.6) that

$$
\begin{aligned}
& \left\|x_{n+1}-x_{n}\right\|+\left\|y_{n+1}-y_{n}\right\| \\
& \leq \theta_{n}\left(\left\|x_{n}-x_{n-1}\right\|+\left\|y_{n}-y_{n-1}\right\|\right) \\
& \quad+k\left(\varepsilon_{n}+\varepsilon_{n-1}\right)+\kappa\left(\varepsilon_{n}+\varepsilon_{n-1}\right)+\left(\left\|d_{n}-d_{n-1}\right\|+\left\|e_{n}-e_{n-1}\right\|\right),
\end{aligned}
$$

where

$$
\theta_{n}=\max \left\{1+\kappa \vartheta_{2}-k\left(1-\theta_{1, n}\right), 1+k \vartheta_{1}-\kappa\left(1-\theta_{2, n}\right)\right\} .
$$

Let

$$
\theta=\max \left\{1+\kappa \vartheta_{2}-k\left(1-\theta_{1}\right), 1+k \vartheta_{1}-\kappa\left(1-\theta_{2}\right)\right\},
$$

where

$$
\begin{aligned}
& \theta_{1}=\nu_{2}+\sqrt[q_{1}]{1-q_{1} \delta_{1}+c_{q_{1}} q_{p}^{q_{1}}}+\frac{\tau_{1}^{q_{1}-1}\left[\left(1-\lambda_{1}\right) \sigma_{1} l_{p}+\rho \lambda_{1} \beta_{1} \xi\right]}{r_{1}-\rho \lambda_{1} m_{1}}, \\
& \theta_{2}=\nu_{1}+\sqrt[q_{2}]{1-q_{2} \delta_{2}+c_{q_{2}} q_{h}^{q_{2}}}+\frac{\tau_{2}^{q_{2}-1}\left[\left(1-\lambda_{2}\right) \sigma_{2} l_{h}+\varrho \lambda_{2} \beta_{2} \zeta\right]}{r_{2}-\varrho \lambda_{2} m_{2}}
\end{aligned}
$$

Then, we know that $\theta_{n} \downarrow \theta$ as $n \rightarrow \infty$.

From the condition (3.4), we know that $0<\theta<1$ and so there exist $n_{0}>0$ and $\theta_{0} \in$ $(\theta, 1)$ such that $\theta_{n} \leq \theta_{0}$ for all $n \geq n_{0}$. Therefore, by (3.7), we have

$$
\begin{aligned}
& \left\|\left(x_{n+1}, y_{n+1}\right)-\left(x_{n}, y_{n}\right)\right\|_{*} \\
& \leq \theta_{0}\left\|\left(x_{n}, y_{n}\right)-\left(x_{n-1}, y_{n-1}\right)\right\|_{*}+\left(\left\|d_{n}-d_{n-1}\right\|+\left\|e_{n}-e_{n-1}\right\|\right) \\
& +k\left(\varepsilon_{n}+\varepsilon_{n-1}\right)+\kappa\left(\varepsilon_{n}+\varepsilon_{n-1}\right) \\
& \leq \theta_{0}^{n-n_{0}}\left\|\left(x_{n_{0}+1}, y_{n_{0}+1}\right)-\left(x_{n_{0}}, y_{n_{0}}\right)\right\|_{*}+\sum_{i=1}^{n-n_{0}} \theta_{0}^{i-1} \varsigma_{n-(i-1)}
\end{aligned}
$$

where $\varsigma_{n}=\left\|d_{n}-d_{n-1}\right\|+\left\|e_{n}-e_{n-1}\right\|+k\left(\epsilon_{n}+\epsilon_{n-1}\right)+\kappa\left(\varepsilon_{n}+\varepsilon_{n-1}\right)$ for all $n \geq n_{0}$. By (3.8), for any $m \geq n>n_{0}$, we have 


$$
\begin{aligned}
& \left\|\left(x_{m}, y_{m}\right)-\left(x_{n}, y_{n}\right)\right\|_{*} \\
& \leq \sum_{j=n}^{m-1}\left(\left\|x_{j+1}-x_{j}\right\|+\left\|y_{j+1}-y_{j}\right\|\right) \\
& \leq \sum_{j=n}^{m-1} \theta_{0}^{j-n_{0}}\left\|\left(x_{n_{0}+1}, y_{n_{0}+1}\right)-\left(x_{n_{0}}, y_{n_{0}}\right)\right\|_{*}+\sum_{j=n}^{m-1} \sum_{i=1}^{j-n_{0}} \theta_{0}^{i-1} \varsigma_{j-(i-1)} .
\end{aligned}
$$

It follows from the hypothesis of Algorithm 3.1, Lemma 2.3 and (3.9) that

$$
\lim _{n \rightarrow \infty}\left\|\left(x^{m}, y^{m}\right)-\left(x^{n}, y^{n}\right)\right\|_{*}=0 .
$$

Hence, $\left\{\left(x^{n}, y^{n}\right)\right\}$ is a Cauchy sequence, i.e., there exists $\left(x^{*}, y^{*}\right) \in \mathcal{B}_{1} \times \mathcal{B}_{2}$ such that $\left(x^{n}, y^{n}\right) \rightarrow\left(x^{*}, y^{*}\right)$ as $n \rightarrow \infty$.

Next, we prove that $u_{n} \rightarrow u^{*} \in S\left(x^{*}\right)$ and $v_{n} \rightarrow v^{*} \in T\left(y^{*}\right)$. In fact, because

$$
\left\|u_{n}-u_{n-1}\right\| \leq\left(1+n^{-1}\right) \hat{\mathbf{H}}\left(S\left(x_{n}\right), S\left(x_{n-1}\right)\right) \leq \xi\left(1+n^{-1}\right)\left\|x_{n}-x_{n-1}\right\|,
$$

it follows that $\left\{u_{n}\right\}$ is also Cauchy sequence in $\mathcal{B}_{1}$. Let $u_{n} \rightarrow u^{*}$. In the sequel, we will show that $u^{*} \in S\left(x^{*}\right)$. Noting $u_{n} \in S\left(x_{n}\right)$, from the results in [22], we have

$$
\begin{aligned}
d\left(u^{*}, S\left(x^{*}\right)\right) & =\inf \left\{\left\|u_{n}-y\right\|: y \in S\left(x^{*}\right)\right\} \leq\left\|u^{*}-u_{n}\right\|+d\left(u_{n}, S\left(x^{*}\right)\right) \\
& \leq\left\|u^{*}-u_{n}\right\|+\hat{\mathbf{H}}\left(S\left(x_{n}\right), S\left(x^{*}\right)\right) \\
& \leq\left\|u^{*}-u_{n}\right\|+\xi\left\|x_{n}-x^{*}\right\| \rightarrow 0 .
\end{aligned}
$$

Hence $d\left(u^{*}, S\left(x^{*}\right)\right)=0$ and therefore $u^{*} \in S\left(x^{*}\right)$. Similarly, we have $v_{n} \rightarrow v^{*} \in T\left(y^{*}\right)$. By continuity and the hypothesis of Algorithm 3.1, we know that $\left(x^{*}, y^{*}, u^{*}, v^{*}\right)$ satisfies the Equation (1.1). This completes the proof.

Remark 3.1. We note that Hilbert space and $L_{p}$ (or $\left.l_{p}\right)(2 \leq p<\infty)$ spaces are 2-uniformly smooth Banach spaces and if $\mathcal{B}_{i}(i=1,2)$ is 2-uniformly smooth Banach space, we can choose constants $v_{i}, \lambda_{i}(i=1,2), \rho$ and $\varrho$ such that (3.4) hold. See, for example, [2-18] and the references therein.

Remark 3.2. Condition (3.4) of Theorem 3.1 holds for some suitable value of constants, for example, $q_{1}=q_{2}=2, c_{2}=1, v_{1}=v_{2}=0.02, \delta_{1}=\delta_{2}=0.3, l_{p}=l_{h}=0.6, \tau_{1}=$ $\tau_{2}=0.05, \lambda_{1}=\lambda_{2}=0.01, \sigma_{1}=\sigma_{2}=0.5, \rho=\varrho=0.1, \beta_{1}=\beta_{2}=0.05, \xi=0.7, \zeta=0.4, r_{1}$ $=r_{2}=0.3, m_{1}=m_{2}=0.2, k=\kappa=0.5, l_{f}=0.2, l_{g}=0.4, \iota_{1}=\iota_{2}=0.05, \varpi_{1}=\varpi_{2}=0.05$ and $\pi_{1}=\pi_{2}=0.2$.

From Theorem 3.1, we have the following results as an application of Theorem 3.1.

Theorem 3.2. Assume that $\mathcal{H}$ is a real Hilbert space and the following conditions hold:

$\left(H_{1}\right) h: \mathcal{H} \rightarrow \mathcal{H}$ is $\delta$-strongly monotone and $l_{h}$-Lipschitz continuous, $f: \mathcal{H} \rightarrow \mathcal{H}$ is $l_{f}$-Lipschitz continuous and $g: \mathcal{H} \rightarrow \mathcal{H}$ is $l_{g}$-Lipschitz continuous;

$\left(H_{2}\right) N: \mathcal{H} \rightarrow \mathcal{H}$ is $\left(\pi_{1}, \iota_{1}\right)$-relaxed cocoercive with respect to $f$ and $\varpi$-Lipschitz continuous, and $\left(\pi_{2}, \iota_{2}\right)$-relaxed cocoercive with respect to $g$;

$\left(H_{3}\right)$ for $i=1,2$ and $n=0,1,2, \ldots, M_{i}^{n}, M_{i}: \mathcal{H} \rightarrow 2^{\mathcal{H}}$ are maximal monotone operators with $M_{i}^{n} \stackrel{A_{1}-G M_{i} \text {; }}{\longrightarrow}$ 
$\left(H_{4}\right)$ there exist positive constants $\rho$ and $\varrho$ such that

$$
\begin{gathered}
\sqrt{1-2 \delta+l_{h}^{2}}<\min \left\{1-\frac{\kappa}{k} \sqrt{l_{g}^{2}-2 \varrho \iota_{2} \varpi^{2}+2 \varrho \pi_{2}+\varrho^{2} \varpi^{2}},\right. \\
\left.1-\frac{k}{\kappa} \sqrt{l_{f}^{2}-2 \rho \iota_{1} \varpi^{2}+2 \rho \pi_{1}+\rho^{2} \varpi^{2}}\right\} .
\end{gathered}
$$

Then, the iterative sequences $\left\{\left(x_{n}, y_{n}\right)\right\}$ generated as follows converges strongly to the common solution $\left(x^{*}, y^{*}\right)$ of the system (1.5):

For any given $\left(x_{0}, y_{0}\right) \in \mathcal{H} \times \mathcal{H}$, define an iterative sequence as follows:

$$
\left\{\begin{array}{l}
x_{n+1}=(1-k) x_{n}+k\left\{x_{n}-h\left(x_{n}\right)+J_{M_{1}^{n}}^{\rho}\left[f\left(y_{n}-\rho N\left(y_{n}\right)\right]\right\}+d_{n}\right. \\
y_{n+1}=(1-\kappa) y_{n}+\kappa\left\{y_{n}-h\left(y_{n}\right)+J_{M_{2}^{n}}^{o}\left[g\left(x_{n}\right)-\varrho N\left(x_{n}\right)\right]\right\}+e_{n}
\end{array}\right.
$$

where $J_{M_{1}^{n}}^{\rho}=\left(I+\rho M_{1}^{n}\right)^{-1}, J_{M_{2}^{n}}^{\varrho}=\left(I+\varrho M_{2}^{n}\right)^{-1}, \rho, \varrho>0, k, \kappa \in(0,1),\left\{d_{n}\right\} \subset \mathcal{H} \quad$ and $\left\{e_{n}\right\} \subset \mathcal{H}$ are two error sequences to take into account a possible inexact computation of the operator points, which satisfy the following conditions:

$$
\lim _{n \rightarrow \infty} d^{n}=\lim _{n \rightarrow \infty} e^{n}=0, \sum_{n=1}^{\infty}\left(\left\|d^{n}-d^{n-1}\right\|+\left\|e^{n}-e^{n-1}\right\|\right)<\infty .
$$

Proof. By the nonexpansivity of the resolvent operators associated withmaximal monotone operators and the proof of Theorem 3.1, one can derivethe result.

Remark 3.3. We note that one can obtain the corresponding results of Theorems 3.1-3.2 when there are problems $(1.1),(1.3)-(1.5)$ with $(H, \eta)$-accretive mappings, $(A$, $\eta)$-monotone operators, $(H, \eta)$-monotone operators, $H$-accretive operators, generalized $m$-accretive operators, maximal $\eta$-monotone operators, $H$-monotone operators, $A$ monotone operators, $\eta$-subdifferential operators or the classical $m$-accretive. The results obtained in this paper improve and generalize the corresponding results of $[2,3,5,12,14,17,18]$ and many other recent works.

\section{Acknowledgements}

This work was supported by the Sichuan Youth Science and Technology Foundation (08ZQ026-008), the Open Foundation of Artificial Intelligence of Key Laboratory of Sichuan Province (2009RZ001), the Scientific Research Fund of Sichuan Provincial Education Department (10ZA136), the Cultivation Project of Sichuan University of Science and Engineering (2011PY01) and the Korea Research Foundation Grant funded by the Korean Government (KRF-2008-313C00050). The authors are grateful to the editor and referee for valuable comments and suggestions.

\section{Author details}

${ }^{1}$ Department of Mathematics, Sichuan University of Science and Engineering, Zigong, 643000, Sichuan, People's Republic of China 2Department of Mathematics Education and the RINS, College of Education, Gyeongsang National University, Chinju 660-701, Korea

\section{Authors' contributions}

FL carried out the proof of convergence of the theorems and gave some examples to show the main results. $\mathrm{H}-\mathrm{YL}$ conceived of the study, and participated in its design and coordination. YJC carried out the check of the manuscript and participated in the design of the study. All authors read and approved the final manuscript.

\section{Competing interests}

The authors declare that they have no competing interests.

Received: 24 April 2011 Accepted: 15 February 2012 Published: 15 February 2012

\section{References}

1. Zeidler, E: Nonlinear functional analysis and its applications. Springer, New Yorkl (1986)

2. Agarwal, RP, Verma, RU: General implicit variational inclusion problems based on A-maximal (m)-relaxed monotonicity (AMRM) frameworks. Appl Math Comput. 215, 367-379 (2009). doi:10.1016/j.amc.2009.04.078 
3. Fang, YP, Huang, NJ: H-accretive operators and resolvent operator technique for solving variatonal inclusionsin Banach spaces. Appl Math Lett. 17(6):647-653 (2004). doi:10.1016/S0893-9659(04)90099-7

4. He, XF, Lou, J, He, Z: Iterative methods for solving variational inclusions in Banach spaces. J Comput Appl Math. 203(1):80-86 (2007). doi:10.1016/j.cam.2006.03.011

5. Jin, MM: Iterative algorithms for a new system of nonlinear vriational inclusions with $(A, \eta)$-accretive mappings in Banach spaces. Comput Math Appl. 54, 579-588 (2007). doi:10.1016/j.camwa.2006.12.030

6. Kazmi, KR, Bhat, Ml: Iterative algorithm for a system of nonlinear variational-like inclusions. Comput Math Appl. 48(12):1929-1935 (2004). doi:10.1016/j.camwa.2004.02.009

7. Lan, HY, Cho, YJ, Verma, RU: On nonlinear relaxed cocoercive variational inclusions involving (A, $\eta$ )-accretive mappings in Banach spaces. Comput Math Appl. 51(9-10):1529-1538 (2006). doi:10.1016/j.camwa.2005.11.036

8. Lan, HY: New proximal algorithms for a class of $(A, \eta)$-accretive variational inclusion problems with non-accretive setvalued mappings. J Appl Math Comput. 25(1-2):255-267 (2007). doi:10.1007/BF02832351

9. Lan, HY, Cai, LC: Variational convergence of a new proximal algorithm for nonlinear general A-monotone operator equation systems in Banach spaces. Nonlinear Anal TMA. 71(12):6194-6201 (2009). doi:10.1016/j.na.2009.06.012

10. Li, HG, Xu, AJ, Jin, MM: A hybrid proximal point three-step algorithm for nonlinear set-valued quasi-variational inclusions system involving (A, n)-accretive mappings. Fixed Point Theory Appl 2010, 24 (2010). Article ID 635382

11. Liou, YC: An iterative algorithm for mixed equilibrium problems and variational inclusions approach to variational inequalities. Fixed Point Theory Appl 2010, 15 (2010). Article ID 56436

12. Noor, MA, Noor, Kl, Al-Said, E: Resolvent iterative methods for solving system of extended general variational inclusions J Inequal Appl 2011, 10 (2011). Article ID 371241. doi:10.1186/1029-242X-2011-10

13. Peng, JW, Zhu, DL, Zheng, XP: Existence of solutions and convergence of a multistep iterative algorithm for a system of variational inclusions with (H, n)-accretive operators. Fixed Point Theory Appl 2007, 20 (2007). Article ID 93678

14. Petrot, N: A resolvent operator technique for approximate solving of generalized system mixed variational inequality and fixed point problems. Appl Math Lett. 23(4):440-445 (2010). doi:10.1016/j.aml.2009.12.001

15. Tan, JF, Chang, SS: Iterative algorithms for finding common solutions to variational inclusion equilibrium and fixed point problems. Fixed Point Theory Appl 2011, 17 (2011). Article ID 915629. doi:10.1186/1687-1812-2011-17

16. Verma, RU: Generalized system for relaxed cocoercive variational inequalities and projection methods. J Optim Theory Appl. 121, 203-210 (2004)

17. Verma, RU: A-monotone nonlinear relaxed cocoercive variational inclusions. Central Eur J Math. 5(2):386-396 (2007). doi:10.2478/s11533-007-0005-5

18. Wang, Z, Wu, C: A system of nonlinear variational inclusions with $(A, \eta)$-monotone mappings. J Inequal Appl 2008, 6 (2008). Article ID 681734

19. Clarke, FH: Optimization and Nonsmooth Analysis. Wiley, New York (1983)

20. Verma, RU: A generalization to variational convergence for operators. Adv Nonlinear var Inequal. 11(2):97-101 (2008)

21. Xu, HK: Inequalities in Banach spaces with applications. Nonlinear Anal. 16(12):1127-1138 (1991). doi:10.1016/0362-546X (91)90200-K

22. Nadler, SB: Muliti-valued contraction mappings. Pac J Math. 30, 475-488 (1969)

doi:10.1186/1687-1812-2012-14

Cite this article as: Li et al:: Graphical approximation of common solutions to generalized nonlinear relaxed cocoercive operator equation systems with $(A, \eta)$-accretive mappings. Fixed Point Theory and Applications 2012 2012:14.

\section{Submit your manuscript to a SpringerOpen ${ }^{\circ}$ journal and benefit from:}

Convenient online submission

- Rigorous peer review

- Immediate publication on acceptance

- Open access: articles freely available online

- High visibility within the field

- Retaining the copyright to your article

Submit your next manuscript at $\gg$ springeropen.com 